ROME1 - 1109/95

ROM2F $/ 95 / 20$

September, 1995

hep-ph/???

\title{
Flavour changing neutral currents and CP violating processes in generalized supersymmetric theories
}

\author{
E. Gabrielli ${ }^{a}$, A. Masiero ${ }^{b}$ and L. Silvestrini ${ }^{c}$ \\ ${ }^{a}$ Dip. di Fisica, Università di Roma "La Sapienza" and \\ INFN, Sezione di Roma, P.le A. Moro 2, I-00185 Roma, Italy. \\ ${ }^{b}$ Dip. di Fisica, Università di Perugia and \\ INFN, Sezione di Perugia, Via Pascoli, I-06100 Perugia, Italy. \\ ${ }^{c}$ Dip. di Fisica, Univ. di Roma "Tor Vergata" and INFN, Sezione di Roma II, \\ Via della Ricerca Scientifica 1, I-00133 Roma, Italy.
}

\begin{abstract}
We consider supersymmetric extensions of the standard model with general non-universal soft breaking terms. We analyse in a modelindependent way the constraints on these terms at the electroweak energy scale coming from gluino mediated flavour (F) changing neutral current and $\mathrm{CP}$-violating processes. We have computed the complete $\Delta F=1$ and $\Delta F=2$ effective hamiltonian for gluino mediated processes, including for the first time the effect of box diagrams in the evaluation of $\epsilon^{\prime} / \epsilon$. We present numerical results for the constraints on these non-universal soft breaking terms for different values of the parameters, extending the analysis also to the leptonic sector. A comparison with previous results in the literature is given.
\end{abstract}


Since the early advent of low energy supersymmetry (SUSY) [1], the flavour changing neutral current (FCNC) tests played a major role in severely constraining the SUSY mass spectrum, in particular the sfermion sector. It is known that the major conclusion was the high degree of degeneracy which is requested in the squark sector [2, 3, 4]. As for CP violating aspects, it turned out that the bound on the electric dipole moment of the neutron prevents any large conspicuous effect due to genuinely SUSY phases, while the SUSY $\mathrm{CP}$ violating contributions related to the usual CKM phase are small in comparison to the standard model (SM) contribution at least in the minimal SUSY model (MSSM) 四. Nowadays there is a renewed interest in both the FCNC and CP issues in SUSY. This is mainly due to some progress that was made in the issue of SUSY breakdown in effective supergravities which emerge as the low energy limit of superstring theories and, consequently, additional information on the soft SUSY breaking terms was gained [5]. It turns out that, somewhat worryingly, the generic pattern of such soft breaking terms does not correspond to the usual universality conditions which are at the basis of the high degeneracy in the sfermion sector. Also the imaginary parts of these soft breaking terms have no a priori reason to be so small.

To test those effective supergravities in their FCNC and $\mathrm{CP}$ violation implications one needs a model independent parameterization of FCNC. A particularly interesting class of these contributions is provided by the gluinoinduced FCNC effects [3, 4]. The most efficient parameterization is obtained in the so-called superKM basis where gluino-quark-squark couplings are diagonal in flavour and all the flavour changing (FC) effects are due to the non-diagonality of the squark mass matrices [6]. As long as the ratio of the off-diagonal entries over an average squark mass remains a small parameter, the first term of the expansion which is obtained by an off-diagonal mass insertion in the squark propagators represents a suitable approximation. The method avoids the specific knowledge of the sfermion mass matrices.

In this letter we present the main results of a new analysis of the full 
SUSY contributions due to gluino or neutralino exchange to the set of FCNC and $\mathrm{CP}$ violating phenomena. The motivation for this study is twofold :

1. the two previous long analyses [7, 8] of this kind, whose results are currently used in the literature, differ quantitatively in several points and a definitive clarification of these controversial aspects is needed;

2. even more important, the constraints coming from $\mathrm{CP}$ violation have so far been only partially included in the analysis.

To be sure, CP violating phenomena were not considered at all in ref. [7], while in ref. [8] the analysis of $\mathrm{CP}$ violation in the $\Delta S=1$ sector takes into account only the superpenguin contributions disregarding SUSY box diagrams. On the contrary, in our analysis we show that SUSY box contributions to $\Delta S=1 \mathrm{CP}$ violation are of the same order as the superpenguins ones and, indeed, the interference of these two classes of contributions leads to results which may differ by one order of magnitude with respect to what was previously found.

We briefly state the ground for our discussion. It has been known for more than ten years now that gluino-quark-squark $(\tilde{g}-q-\tilde{q})$ vertices can exhibit flavour change [3, 4 . The point is that in general the $q$ and $\tilde{q}$ mass matrices are not simultaneously diagonalizable. This might be due to the initial conditions: SUSY breaking terms may yield contributions which are not universal [5], i.e. they are not proportional to the unit matrix in flavour space. Otherwise, even starting with universal mass contribution to sfermions in the SUSY soft breaking sector, renormalization effects from the starting point, i.e. the scale of supergravity breaking, down to the Fermi scale can bring about a misalignment between $q$ and $\tilde{q}$ mass matrices [3, 4]. This latter situation is what we encounter in the minimal SUSY standard model. For instance, consider the mass matrix squared of the scalar partner of the left-handed down-quarks $d_{L}$. At the scale of supergravity breaking this matrix consists of the SUSY conserving contribution $m_{d} m_{d}^{\dagger}$ (where $m_{d}$ denotes 
the down quark mass matrix) and the SUSY breaking universal contribution $\tilde{m}^{2} \mathbb{I}$. However, the term $h_{u} Q H u^{c}$ of the superpotential generates a logarithmically divergent contribution which is proportional to $h_{u} h_{u}^{\dagger}$ and, hence, to $m_{u} m_{u}^{\dagger}\left(m_{u}\right.$ being the up-quark mass matrix). Hence the resulting $\tilde{d}_{L}$ mass matrix squared at the Fermi scale is:

$$
m_{\tilde{d}_{L} \tilde{d}_{L}}^{2}=m_{d} m_{d}^{\dagger}+\tilde{m}^{2} \mathbb{I}+c m_{u} m_{u}^{\dagger}
$$

Clearly the $\left(\tilde{g}-d_{L}-\tilde{d}_{L}\right)$ couplings are no longer flavour diagonal given the presence of the last term in the r.h.s. of eq. (1). The above mentioned massinsertion approximation results from choosing the so-called super-KM matrix where one takes a basis for the $\tilde{d}_{L}$ so that the $\tilde{g}-d_{L}-\tilde{d}_{L}$ are flavour diagonal and the FCNC effects are accounted for by flavour changing mass insertions in the $\tilde{q}$ propagators. From eq. (四) it is easy to realize that the mass insertion needed to accomplish the transition from $\tilde{d}_{i L}$ to $\tilde{d}_{j L}(i, j$ flavour indices) is given by:

$$
\left(\Delta_{L L}^{d}\right)_{i j}=c\left[K\left(m_{u}^{\text {diag }}\right)^{2} K^{\dagger}\right]_{i j}
$$

where $K$ is the Cabibbo-Kobayashi-Maskawa matrix and $m_{u}^{\text {diag }}$ denotes the diagonalized up-quark mass matrix. In the following, with the notation $\left(\Delta_{A B}^{q}\right)_{i j}$, we mean the mass insertion needed for a transition from a squark $\tilde{q}_{i A}$ to $\tilde{q}_{j B}$ with $A=(L, R)$ and $B=(L, R)$. Actually, as particularly emphasized in ref. [8], the above expression for $\left(\Delta_{L L}^{d}\right)_{i j}$ may be somewhat misleading since one might think that the term $c$ in the r.h.s. of eq. (2) is a constant (i.e. independent of the SUSY breaking scale) or at most depends logarithmically on it. On the contrary, the one-loop RGE's show that $c$ depends quadratically on that scale. Since also the average squark mass is proportional to this scale, the meaningful parameter for our mass insertion approximation is the dimensionless quantity $\delta=\Delta / \tilde{m}^{2}$, where $\tilde{m}$ denotes at the same time the average squark mass and the typical SUSY breaking scale. This observation is of utmost relevance if one wants to understand the scaling of the SUSY contribution to FCNC with increasing squark masses. 
The powers of $\tilde{m}$ in the denominator which are present to compensate for $\Delta$ mass insertions in the numerator do not have to be considered if also $\Delta$ is proportional to $\tilde{m}^{2}$. This justifies why gluino-induced FCNC SUSY contributions remain still sizeable even for $\tilde{q}$ masses above $1 \mathrm{TeV}$ [8], as we will see in what follows.

Two comments are in order before giving our results. There are three classes of sfermion mixings according to the helicity of their fermionic partners: $\Delta_{L L}, \Delta_{R R}, \Delta_{L R}$. In the MSSM there is a sharp hierarchy among them [7]. Only $\Delta_{L L}$ appears as a simple mass insertion since the FC effect is related to the property of the $\tilde{q}_{L}$ to sit in $\mathrm{SU}(2)$ doublets, $\Delta_{L R}$ results from a FC $\Delta_{L L}$ insertion followed by a $\Delta_{R R}$ flavour conserving insertion. Finally a $\Delta_{R R}$ FC contribution would require three mass insertions since only $\Delta_{L L}$ can yield a FC. Hence $\left(\Delta_{L L}\right)_{i j}>>\left(\Delta_{L R}\right)_{i j}>>\left(\Delta_{R R}\right)_{i j}$ with $i \neq j$ in the MSSM. However, it should be clear from the above sketched argument of the source of FC in the $\tilde{q}$ propagators in the MSSM, that this conclusion is quite model-dependent. In particular, if FC effects are produced by "initial" conditions one cannot make any general statement on the relative size of the three contributions $\Delta_{L L}, \Delta_{L R}, \Delta_{R R}$.

The second observation is related to the appearance of the CKM elements in the expressions of the $\Delta_{L L}$ in the MSSM (eq. (2))). In the gluino-induced FC contributions one obtains a GIM suppression mechanism which is entirely analogue of what occurred in $\mathrm{W}$-mediated FCNC effects in the SM, in particular with the same CKM angles and phase. This is crucial in understanding the smallness of the SUSY contributions both in the FCNC and CP violating phenomena. In particular, the smallness of the angles connecting transitions between first and third generation is at the basis of the smallness also of the gluino-induced SUSY contributions to CP violation in the Kaon system (we are assuming here that the only source of CP violation in the MSSM is the CKM phase). Also this property is very specific of the MSSM structure, in particular of the universality of its soft breaking terms (see the second term 


\begin{tabular}{||c|c|c|c||}
\hline \hline$x$ & $\sqrt{\mid \operatorname{Re}\left(\delta_{12}^{d}\right)_{L L}^{2}} \mid$ & $\sqrt{\left|\operatorname{Re}\left(\delta_{12}^{d}\right)_{L R}^{2}\right|}$ & $\sqrt{\left|\operatorname{Re}\left(\delta_{12}^{d}\right)_{L L}\left(\delta_{12}^{d}\right)_{R R}\right|}$ \\
\hline 0.3 & $1.9 \times 10^{-2}$ & $7.9 \times 10^{-3}$ & $2.5 \times 10^{-3}$ \\
1.0 & $4.0 \times 10^{-2}$ & $4.4 \times 10^{-3}$ & $2.8 \times 10^{-3}$ \\
4.0 & $9.3 \times 10^{-2}$ & $5.3 \times 10^{-3}$ & $4.0 \times 10^{-3}$ \\
\hline \hline$x$ & $\sqrt{\left|\operatorname{Re}\left(\delta_{13}^{d}\right)_{L L}^{2}\right|}$ & $\sqrt{\left|\operatorname{Re}\left(\delta_{13}^{d}\right)_{L R}^{2}\right|}$ & $\sqrt{\left|\operatorname{Re}\left(\delta_{13}^{d}\right)_{L L}\left(\delta_{13}^{d}\right)_{R R}\right|}$ \\
\hline 0.3 & $4.6 \times 10^{-2}$ & $5.6 \times 10^{-2}$ & $1.6 \times 10^{-2}$ \\
1.0 & $9.8 \times 10^{-2}$ & $3.3 \times 10^{-2}$ & $1.8 \times 10^{-2}$ \\
4.0 & $2.3 \times 10^{-1}$ & $3.6 \times 10^{-2}$ & $2.5 \times 10^{-2}$ \\
\hline \hline$x$ & $\sqrt{\left|\operatorname{Re}\left(\delta_{12}^{u}\right)_{L L}^{2}\right|}$ & $\sqrt{\mid \operatorname{Re}\left(\delta_{12}^{u}\right)_{L R}^{2}}$ & $\sqrt{\left|\operatorname{Re}\left(\delta_{12}^{u}\right)_{L L}\left(\delta_{12}^{u}\right)_{R R}\right|}$ \\
\hline 0.3 & $4.7 \times 10^{-2}$ & $6.3 \times 10^{-2}$ & $1.6 \times 10^{-2}$ \\
1.0 & $1.0 \times 10^{-1}$ & $3.1 \times 10^{-2}$ & $1.7 \times 10^{-2}$ \\
4.0 & $2.4 \times 10^{-1}$ & $3.5 \times 10^{-2}$ & $2.5 \times 10^{-2}$ \\
\hline \hline
\end{tabular}

Table 1: Limits on $\operatorname{Re}\left(\delta_{i j}\right)_{A B}\left(\delta_{i j}\right)_{C D}$, with $A, B, C, D=(L, R)$, for a squark mass $\tilde{m}=500 \mathrm{GeV}$ and for different values of $x=m_{\tilde{g}}^{2} / \tilde{m}^{2}$.

in the r.h.s. of eq. (11)).

We now come to the results of our analysis concerning the terms $\left(\Delta_{L L}\right)_{i j}$, $\left(\Delta_{L R}\right)_{i j}$ and $\left(\Delta_{R R}\right)_{i j}$ in the $\mathrm{u}$ - and d-sectors. In the following we consider the case in which $\left(\Delta_{L R}\right)_{i j} \simeq\left(\Delta_{R L}\right)_{i j}$. We will comment later on the analogous contributions in the charged lepton sector.

First we consider the $\mathrm{CP}$ conserving $\mathrm{FCNC}$ processes. In the down sector the $\Delta_{i j}$ mass insertions are bounded by the $K-\bar{K}$ mass difference $\left(\delta_{12}\right)$, the $B_{d}-\bar{B}_{d}$ mixing $\left(\delta_{13}\right)$ and the branching ratio $\operatorname{BR}(b \rightarrow s+\gamma)\left(\delta_{23}\right)$, while the only available bound in the up-sector concerns $\Delta_{12}$ from $D-\bar{D}$ mixing. We report our results in tables 1 and 2 for an average $\tilde{q}$ mass of $500 \mathrm{GeV}$ 


\begin{tabular}{||c|c|c||}
\hline \hline$x$ & $\left|\left(\delta_{23}^{d}\right)_{L L}\right|$ & $\left|\left(\delta_{23}^{d}\right)_{L R}\right|$ \\
\hline 0.3 & 4.4 & $1.3 \times 10^{-2}$ \\
1.0 & 8.2 & $1.6 \times 10^{-2}$ \\
4.0 & 26 & $3.0 \times 10^{-2}$ \\
\hline \hline
\end{tabular}

Table 2: Limits on the $\left|\delta_{23}^{d}\right|$ from $b \rightarrow s \gamma$ decay for a squark mass $\tilde{m}=$ $500 \mathrm{GeV}$ and for different values of $x=m_{\tilde{g}}^{2} / \tilde{m}^{2}$.

and for different values of $x=m_{\tilde{g}}^{2} / \tilde{m}^{2}$, where $m_{\tilde{g}}$ is the gluino mass. Table 2 shows that the decay $(b \rightarrow s+\gamma)$ does not limit the $\delta_{L L}$ insertion for a SUSY breaking of $\mathrm{O}(500 \mathrm{GeV})$. Indeed, even taking $m_{\tilde{q}}=100 \mathrm{GeV}$, the term $\left(\delta_{23}\right)_{L L}$ is only marginally limited $\left(\left(\Delta_{L L}\right)_{23}<0.3\right.$ for $\left.x=1\right)$. Obviously, $\left(\delta_{23}^{d}\right)_{L R}$ is much more constrained since with a $\Delta_{L R} \mathrm{FC}$ mass insertion the helicity flip needed for $(b \rightarrow s+\gamma)$ is realized in the gluino internal line and so this contribution has an amplitude enhancement of a factor $m_{\tilde{g}} / m_{b}$ over the previous case with $\Delta_{L L}$.

Concerning the calculation of the bounds in tables 1 and 2 we find some discrepancies with previous results quoted in the literature. In eqs. (3.2 a and c) of ref. [7] the terms proportional to the function $M(x)$ must be multiplied by the coefficient $(-1 / 2)$, while in eq. (3.2 b) the function $G(x)$ must be multiplied by $(-1)$. In eq. (4.2) of ref. [8], the terms proportional to $\Delta_{L L} \Delta_{R R} \cdot m_{K} /\left(m_{s}+m_{d}\right)$ and $\left(\Delta_{R L}\right)^{2} \cdot m_{K} /\left(m_{s}+m_{d}\right)$ must be multiplied by $(-1)$ and $(-11 / 18)$ respectively, while the major difference concerns the contribution to $\Delta m_{K}$ proportional to $\Delta_{L R} \Delta_{R L}$ mass insertion given by:

$$
\Delta m_{K}=\frac{\alpha_{s}^{2}}{216 M_{\tilde{q}}^{2}} \frac{2}{3} f_{K}^{2} m_{K} \frac{\delta \tilde{m}_{d_{L} s_{R}}^{2} \delta \tilde{m}_{d_{R} s_{L}}^{2}}{M_{\tilde{q}}^{4}} \tilde{f}_{6}(x)\left[84+144\left(\frac{m_{K}}{m_{s}+m_{d}}\right)^{2}\right]
$$

where we have used the same notation of ref. [8] for comparison. The com- 
plete expression for $\Delta m_{K}$ can be found in ref. [10, together with more details on the analysis.

A similar analysis can be performed in the leptonic sector where the masses $\tilde{m}$ and $m_{\tilde{g}}$ are replaced by the average slepton mass and the photino mass $m_{\tilde{\gamma}}$ respectively. A clear but important point to be stressed is that the severe bounds that we provide on the $\delta_{L L}$ and $\delta_{L R}$ mass insertions in the leptonic sector and the consequent need for high degeneracy of charged sleptons, only apply if separate lepton numbers are violated. It is well known that in the MSSM the lepton numbers $L_{e}, L_{\mu}$ and $L_{\tau}$ are separately conserved because of the diagonality of the soft breaking terms and the masslessness of neutrinos. If at least one of this two properties is not present one can have partial lepton number violation. A particularly interesting example is the case where neutrinos acquire a mass through a see-saw mechanism (for its SUSY version and the implications for FCNC see [9]). In table 3 we exhibit the bounds on $\delta_{L L}^{l}$ and $\delta_{L R}^{l}$ coming from the limits on $\mu \rightarrow e \gamma, \tau \rightarrow e \gamma$ and $\tau \rightarrow \mu \gamma$, for a slepton mass of $\mathrm{O}(100 \mathrm{GeV})$ and for different values of $x=m_{\tilde{\gamma}}^{2} / \tilde{m}^{2}$. Our results confirm those obtained in refs [0, \&].

We tackle now the subject of one-loop CP violating contributions through gluino exchange. As for the $\Delta S=2$ transitions, the corresponding bounds on $\operatorname{Im}\left(\delta_{12}^{d}\right)_{L L}^{2}$ and $\operatorname{Im}\left(\delta_{12}^{d}\right)_{L R}^{2}$ are readily obtained from those derived for the real parts from $\Delta m_{K}$. What is actually new in our analysis with respect to ref. [8], but more generally, with respect to all previous works dealing with gluino-induced CP violation, is our treatment of the SUSY contributions to direct $\mathrm{CP}$ violation in $\Delta S=1$ processes. Indeed only superpenguins were considered to be relevant for $\epsilon^{\prime}$, while we obtain that also box diagrams with gluinos exchange (see fig.1) give a sizeable contributions with relevant interference effects with the superpenguins.

The contribution to the effective Hamiltonian for $\Delta S=1$ transitions 


\begin{tabular}{||c|c|c||}
\hline \hline$x$ & $\left|\left(\delta_{12}^{l}\right)_{L L}\right|$ & $\left|\left(\delta_{12}^{l}\right)_{L R}\right|$ \\
\hline 0.3 & $4.1 \times 10^{-3}$ & $1.4 \times 10^{-6}$ \\
1.0 & $7.7 \times 10^{-3}$ & $1.7 \times 10^{-6}$ \\
5.0 & $3.2 \times 10^{-2}$ & $3.8 \times 10^{-6}$ \\
\hline \hline & & \\
$x$ & $\left|\left(\delta_{13}^{l}\right)_{L L}\right|$ & $\left|\left(\delta_{13}^{l}\right)_{L R}\right|$ \\
& & \\
\hline 0.3 & 15 & $8.9 \times 10^{-2}$ \\
1.0 & 29 & $1.1 \times 10^{-1}$ \\
5.0 & $1.2 \times 10^{2}$ & $2.4 \times 10^{-1}$ \\
\hline \hline & & \\
$x$ & $\left|\left(\delta_{23}^{l}\right)_{L L}\right|$ & $\left|\left(\delta_{23}^{l}\right)_{L R}\right|$ \\
\hline 0.3 & 2.8 & $1.7 \times 10^{-2}$ \\
1.0 & 5.3 & $2.0 \times 10^{-2}$ \\
5.0 & 22 & $4.4 \times 10^{-2}$ \\
\hline \hline
\end{tabular}

Table 3: Limits on the $\left|\delta_{i j}^{d}\right|$ from $l_{j} \rightarrow l_{i} \gamma$ lepton decay for a slepton mass $\tilde{m}=100 \mathrm{GeV}$ and for different values of $x=m_{\tilde{\gamma}}^{2} / \tilde{m}^{2}$. 
given by the gluino penguins and box diagrams can be written as

$$
\mathcal{H}_{\rceil\{\{}=\sum_{\rangle=\ni, 1}\left\{\mathcal{C}_{\rangle} \mathcal{O}_{\rangle}+\mathcal{E}_{\rangle} \mathcal{O}_{\rangle}\right\}
$$

where we have chosen a basis of local four-fermion operators $O_{i}$ :

$$
\begin{aligned}
& O_{3}=\left(\bar{d}_{L}^{\alpha} \gamma^{\mu} s_{L}^{\alpha}\right) \sum_{q=u, d, s}\left(\bar{q}_{L}^{\beta} \gamma_{\mu} q_{L}^{\beta}\right) \\
& O_{4}=\left(\bar{d}_{L}^{\alpha} \gamma^{\mu} s_{L}^{\beta}\right) \sum_{q=u, d, s}\left(\bar{q}_{L}^{\beta} \gamma_{\mu} q_{L}^{\alpha}\right) \\
& O_{5}=\left(\bar{d}_{L}^{\alpha} \gamma^{\mu} s_{L}^{\alpha}\right) \sum_{q=u, d, s}\left(\bar{q}_{R}^{\beta} \gamma_{\mu} q_{R}^{\beta}\right) \\
& O_{6}=\left(\bar{d}_{L}^{\alpha} \gamma^{\mu} s_{L}^{\beta}\right) \sum_{q=u, d, s}\left(\bar{q}_{R}^{\beta} \gamma_{\mu} q_{R}^{\alpha}\right) \\
& O_{7}=\frac{g}{8 \pi^{2}} \bar{d}_{R}^{\alpha} \sigma^{\mu \nu} t_{\alpha \beta}^{A} s_{L}^{\beta} G_{\mu \nu}^{A}
\end{aligned}
$$

where the operators $\tilde{O}_{i}$ can be obtained from $O_{i}$ by the exchange $L \leftrightarrow R$. Here $q_{R, L}=\frac{\left(1 \pm \gamma_{5}\right)}{2} q, \sigma^{\mu \nu}=\frac{i}{2}\left[\gamma^{\mu}, \gamma^{\nu}\right], \alpha$ and $\beta$ are colour indices and $g$ is the strong coupling. The colour matrices normalization is $\operatorname{Tr}\left(t^{A} t^{B}\right)=\delta^{A B} / 2$.

The Wilson coefficients are given by:

$$
\begin{aligned}
C_{3} & =\frac{\alpha_{s}^{2}}{\tilde{m}^{2}}\left(\delta_{12}^{d}\right)_{L L}\left(-\frac{1}{9} \mathrm{~B}_{1}(x)-\frac{5}{9} \mathrm{~B}_{2}(x)-\frac{1}{18} \mathrm{P}_{1}(x)-\frac{1}{2} \mathrm{P}_{2}(x)\right) \\
C_{4} & =\frac{\alpha_{s}^{2}}{\tilde{m}^{2}}\left(\delta_{12}^{d}\right)_{L L}\left(-\frac{7}{3} \mathrm{~B}_{1}(x)+\frac{1}{3} \mathrm{~B}_{2}(x)+\frac{1}{6} \mathrm{P}_{1}(x)+\frac{3}{2} \mathrm{P}_{2}(x)\right) \\
C_{5} & =\frac{\alpha_{s}^{2}}{\tilde{m}^{2}}\left(\delta_{12}^{d}\right)_{L L}\left(\frac{10}{9} \mathrm{~B}_{1}(x)+\frac{1}{18} \mathrm{~B}_{2}(x)-\frac{1}{18} \mathrm{P}_{1}(x)-\frac{1}{2} \mathrm{P}_{2}(x)\right) \\
C_{6} & =\frac{\alpha_{s}^{2}}{\tilde{m}^{2}}\left(\delta_{12}^{d}\right)_{L L}\left(-\frac{2}{3} \mathrm{~B}_{1}(x)+\frac{7}{6} \mathrm{~B}_{2}(x)+\frac{1}{6} \mathrm{P}_{1}(x)+\frac{3}{2} \mathrm{P}_{2}(x)\right) \\
C_{7} & =\frac{\alpha_{s} \pi}{\tilde{m}^{2}}\left[\left(\delta_{12}^{d}\right)_{R R} m_{s}\left(-\frac{1}{3} \mathrm{M}_{3}(x)-3 \mathrm{M}_{4}(x)\right)\right. \\
& \left.+\left(\delta_{12}^{d}\right)_{R L} m_{\tilde{g}}\left(-\frac{1}{3} \mathrm{M}_{1}(x)-3 \mathrm{M}_{2}(x)\right)\right]
\end{aligned}
$$

where again the coefficients $\tilde{C}_{i}$ can be obtained from the $C_{i}$ just by the exchange $L \leftrightarrow R, x=m_{\tilde{g}}^{2} / \tilde{m}^{2}$, and $m_{s}$ is the mass of the strange quark. 


\begin{tabular}{||c|c|c|c|c|c||}
\hline \hline$x$ & $\left|\mathrm{I}_{m}\left(\delta_{12}^{d}\right)_{L L}\right|$ & $\left|\mathrm{I}_{m}\left(\delta_{12}^{d}\right)_{L R}\right|$ & $\sqrt{\left|\mathrm{I}_{m}\left(\delta_{12}^{d}\right)_{L L}^{2}\right|}$ & $\sqrt{\left|\mathrm{I}_{m}\left(\delta_{12}^{d}\right)_{L R}^{2}\right|}$ & $\sqrt{\left|\mathrm{I}_{m}\left(\delta_{12}^{d}\right)_{L L}\left(\delta_{12}^{d}\right)_{R R}\right|}$ \\
\hline 0.3 & $1.0 \times 10^{-1}$ & $1.1 \times 10^{-5}$ & $1.5 \times 10^{-3}$ & $6.3 \times 10^{-4}$ & $2.0 \times 10^{-4}$ \\
1.0 & $4.8 \times 10^{-1}$ & $2.0 \times 10^{-5}$ & $3.2 \times 10^{-3}$ & $3.5 \times 10^{-4}$ & $2.2 \times 10^{-4}$ \\
4.0 & $2.6 \times 10^{-1}$ & $6.3 \times 10^{-5}$ & $7.5 \times 10^{-3}$ & $4.2 \times 10^{-4}$ & $3.2 \times 10^{-4}$ \\
\hline \hline
\end{tabular}

Table 4: Limits on $\operatorname{Im}\left(\delta_{12}^{d}\right)_{A B}$ and on $\operatorname{Im}\left(\delta_{12}^{d}\right)_{A B}\left(\delta_{12}^{d}\right)_{C D}$, with $A, B, C, D=$ $(L, R)$, for a squark mass $\tilde{m}=500 \mathrm{GeV}$ and for different values of $x=m_{\tilde{g}}^{2} / \tilde{m}^{2}$.

The functions $B_{i}(x)$ which result from the calculation of the box diagrams are given by:

$$
\begin{aligned}
& \mathrm{B}_{1}(x)=\frac{1+4 x-5 x^{2}+4 x \ln (x)+2 x^{2} \ln (x)}{8(1-x)^{4}} \\
& \mathrm{~B}_{2}(x)=x \frac{5-4 x-x^{2}+2 \ln (x)+4 x \ln (x)}{2(1-x)^{4}}
\end{aligned}
$$

while the functions $P_{i}(x)$ and $M_{i}(x)$ of the superpenguins can be derived from ref. [8]. In particular our results for the superpenguins coincide with those of ref. [8].

In table 4 we give the bounds on the imaginary parts of $\left(\delta_{12}^{d}\right)_{L L},\left(\delta_{12}^{d}\right)_{L R}$ (from $\Delta S=1$ transitions), $\left(\delta_{12}^{d}\right)_{L L}^{2},\left(\delta_{12}^{d}\right)_{L R}^{2}$ and $\left(\delta_{12}^{d}\right)_{L L}\left(\delta_{12}^{d}\right)_{R R}$ (from $\Delta S=2$ transitions) for an average squark mass $\tilde{m}=500 \mathrm{GeV}$. In figs. 2 and 3 we exhibit the behaviour of the upper bound of $\operatorname{Im}\left(\delta_{12}\right)_{L L}$ and $\operatorname{Im}\left(\delta_{12}\right)_{L R}$ as a function of $x=m_{\tilde{g}}^{2} / \tilde{m}^{2}$ and for $\tilde{m}=100 \mathrm{GeV}$. These bounds are obtained for an upper limit of $\epsilon^{\prime} / \epsilon=10^{-3}$. The effect of the interference between penguin and box contributions is particularly severe for certain ranges of $x$. For instance, this is what occurs in the proximity of $x=1$ for the bounds in $\operatorname{Im}\left(\delta_{12}\right)_{L L}$ (fig. 2). The complete expression for the separate box and penguin contributions to the $\Delta S=1$ effective hamiltonian will be given elsewhere [11]. 
From the results in table 1 it is clear that, if one wishes to obtain a sizeable contribution to $\epsilon^{\prime}$ from one-loop gluino exchange, then $\operatorname{Im}\left(\delta_{12}\right)_{L L}$ should be of $\mathrm{O}\left(10^{-1}\right)$. Moreover, to respect the bound $\operatorname{Im}\left(\delta_{12}\right)_{L L}^{2}<10^{-6}$ from $\epsilon, \operatorname{Re}\left(\delta_{12}\right)_{L L}$ should be extremely small. Hence, unless $\left(\delta_{12}\right)_{L L}$ is essentially imaginary and taking into account the bound from $\epsilon$, no large contribution to $\epsilon^{\prime}$ can arise from $\left(\delta_{12}\right)_{L L}$ mass insertion. On the other hand, it is also apparent from table 4 that a conspicuous contribution to $\epsilon^{\prime}$, coming from $\operatorname{Im}\left(\delta_{12}\right)_{L R}$, can arise in models with sizeable $\delta_{L R}$ mass insertions without conflicting with the bound on this quantity coming from $\epsilon$.

Hence, although quantitatively the inclusion of the $\Delta S=1$ box contributions changes the results of ref. [8], we confirm the main qualitative remark that those authors make, i.e. that SUSY models with predominantly $\delta_{L L}$ or $\delta_{R R}$ contributions to CP violation (such as the MSSM) are likely to be superweak, while models with sizeable contribution to CP violation through $\delta_{L R}$ mass insertions tend to be milliweak. The implications of these considerations on SUSY contributions to CP violation in models with non-universal soft breaking terms are presently under study [11].

\section{Acknowledgements}

We would like to thank G. Giudice for interesting discussions and the physics department of Padova for its kind hospitality. One of us, L.S., would like to acknowledge useful discussions with R. Petronzio.

\section{References}

[1] For a phenomenologically oriented review, see:

P. Fayet and S. Ferrara, Phys. Rep. 32C (1977) 249;

H.P. Nilles, Phys. Rep. 110C (1984) 1.

For spontaneously broken $\mathrm{N}=1$ supergravity, see: 
E. Cremmer, S. Ferrara, L. Girardello and A. Van Proeyen, Nucl. Phys. B 212 (1983) 413 and references therein.

[2] J. Ellis and D.V. Nanopoulos, Phys. Lett. B 110 (1982) 44;

R. Barbieri and R. Gatto, Phys. Lett. B 110 (1982) 211.

[3] M.J. Duncan, Nucl. Phys. B 221 (1983) 285;

J.F. Donoghue, H.P. Nilles and D. Wyler, Phys. Lett. B 128 (1983) 55;

A. Bouquet, J. Kaplan and C.A. Savoy, Phys. Lett. B 148 (1984) 69;

M.J. Duncan and J. Trampetic, Phys. Lett. B 134 (1984) 439;

T. Inami and C.S. Lim, Nucl. Phys. B 207 (1982) 533;

J.-M. Frère and M.B. Gavela, Phys. Lett. B 132 (1983) 107;

E. Franco and M. Mangano, Phys. Lett. B 135 (1984) 445;

S. Bertolini, F. Borzumati and A. Masiero, Phys. Lett. B 192 (1987) 437;

G. Altarelli and P.J. Franzini, Zeit. für Physik C 37 (1988) 271;

S. Bertolini, F. Borzumati and A. Masiero, Phys. Lett. B 194 (1987) 545,

( Erratum, Phys. Lett. B 198 (1987) 590);

T.M. Aliev and M.I. Dobroliubov, Phys. Lett. B 237 (1990) 573;

S. Bertolini, F. Borzumati, A. Masiero and G. Ridolfi, Nucl. Phys. B 353 (1991) 591;

J. Hisano, T. Moroi, K. Tobe, M. Yamaguchi and T. Yanagida, TU-476, hep-ph/ 9501407.

[4] B.A. Campbell, Phys. Rev. D 28 (1983) 209;

F. del Aguila, J.A. Grifols, A. Mendez, D.V. Nanopoulos and M. Srednicki, Phys. Lett. B 129 (1983) 77;

J.-M. Gerard, W. Grimus, A. Raychaudhuri and G. Zoupanos, Phys. Lett. B 140 (1984) 349;

J.-M. Gerard, W. Grimus, A. Masiero, D.V. Nanopoulos and A. Raychaudhuri, Phys. Lett. B 141 (1984) 79;

P. Langacher and R. Sathiapalan, Phys. Lett. B 144 (1984) 401;

J.-M. Gerard, W. Grimus and A. Raychaudhuri, Phys. Lett. B 145 (1984) 400;

M. Dugan, B. Grinstein and L. Hall, Nucl. Phys. B 255 (1985) 413;

L.J. Hall, V.A. Kostelecky and S. Raby, Nucl. Phys. B 267 (1986) 415;

J.S. Hagelin and L.S. Littenberg, Prog. Part. Nucl. Phys. 23 (1989) 1;

E. Gabrielli and G. Giudice, Nucl. Phys. B 433 (1995) 3.

[5] L. Ibanez and D. Lüst, Nucl. Phys. B 382 (1992) 305;

V. Kaplunovsky and J. Louis, Phys. Lett. B 306 (1993) 269; 
R. Barbieri, J. Louis and M. Moretti, Phys. Lett. B 312 (1993) 451, ( Erratum, Phys. Lett. B 316 (1993) 632);

B. de Carlos, J.A. Casas and C. Muñoz, Phys. Lett. B 299 (1993) 234; Nucl. Phys. B 399 (1993) 623;

A. Brignole, L.E. Ibanez and C. Muñoz, Nucl. Phys. B 422 (1994) 125, ( Erratum, Nucl. Phys. B 436 (1995) 747);

Y. Nir and N. Seiberg, Phys. Lett. B 309 (1993) 337;

M. Dine, R. Leigh and A. Kagan, Phys. Rev. D 48 (1993) 4269;

A. Lleyda and C. Muñoz, Phys. Lett. B 317 (1993) 82;

N. Polonski and A. Pomarol, Phys. Rev. Lett. 73 (1994) 2292;

D. Matalliotakis and H.P. Nilles, Nucl. Phys. B 435 (1995) 115;

M. Olechowski and S. Pokorski, Phys. Lett. B 344 (1995) 201;

D. Choudhury, F. Eberlein, A. Konig, J. Louis and S. Pokorski, Phys. Lett. B 342 (1995) 180.

[6] L.J. Hall, V.A. Kostelecky and S. Raby, in ref. [4].

[7] F. Gabbiani and A. Masiero, Nucl. Phys. B 322 (1989) 235.

[8] J.S. Hagelin, S. Kelley and T. Tanaka, Nucl. Phys. B 415 (1994) 293.

[9] F. Borzumati and A. Masiero, Phys. Rev. Lett. 57 (1986) 961.

[10] E. Gabrielli, A. Masiero and L. Silvestrini, Proc. SUSY '95, Ecole Polytechnique, Palaiseau, May 1995, Rome1 - 1113/95, ROM2F/95/23.

[11] F. Gabbiani, E. Gabrielli, A. Masiero and L. Silvestrini, in preparation. 


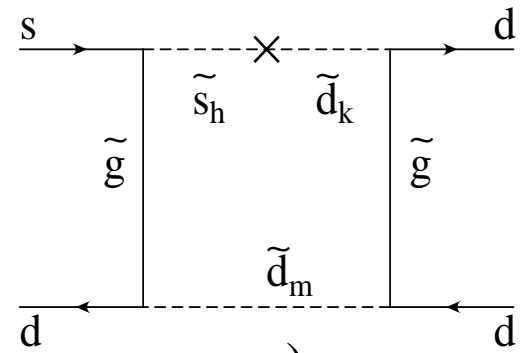

a)

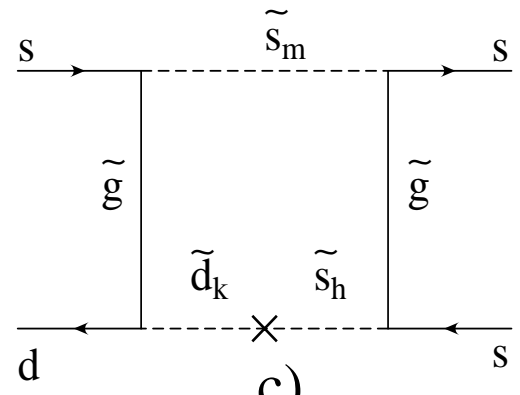

c)

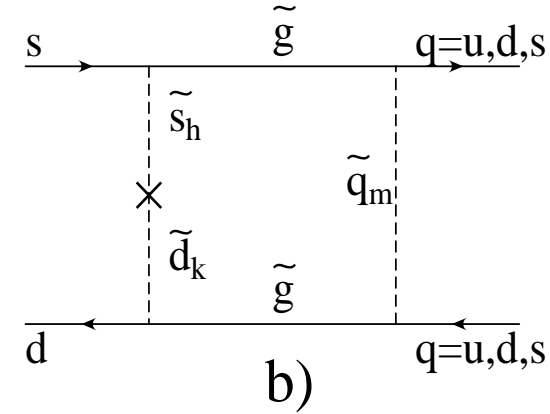

b) 


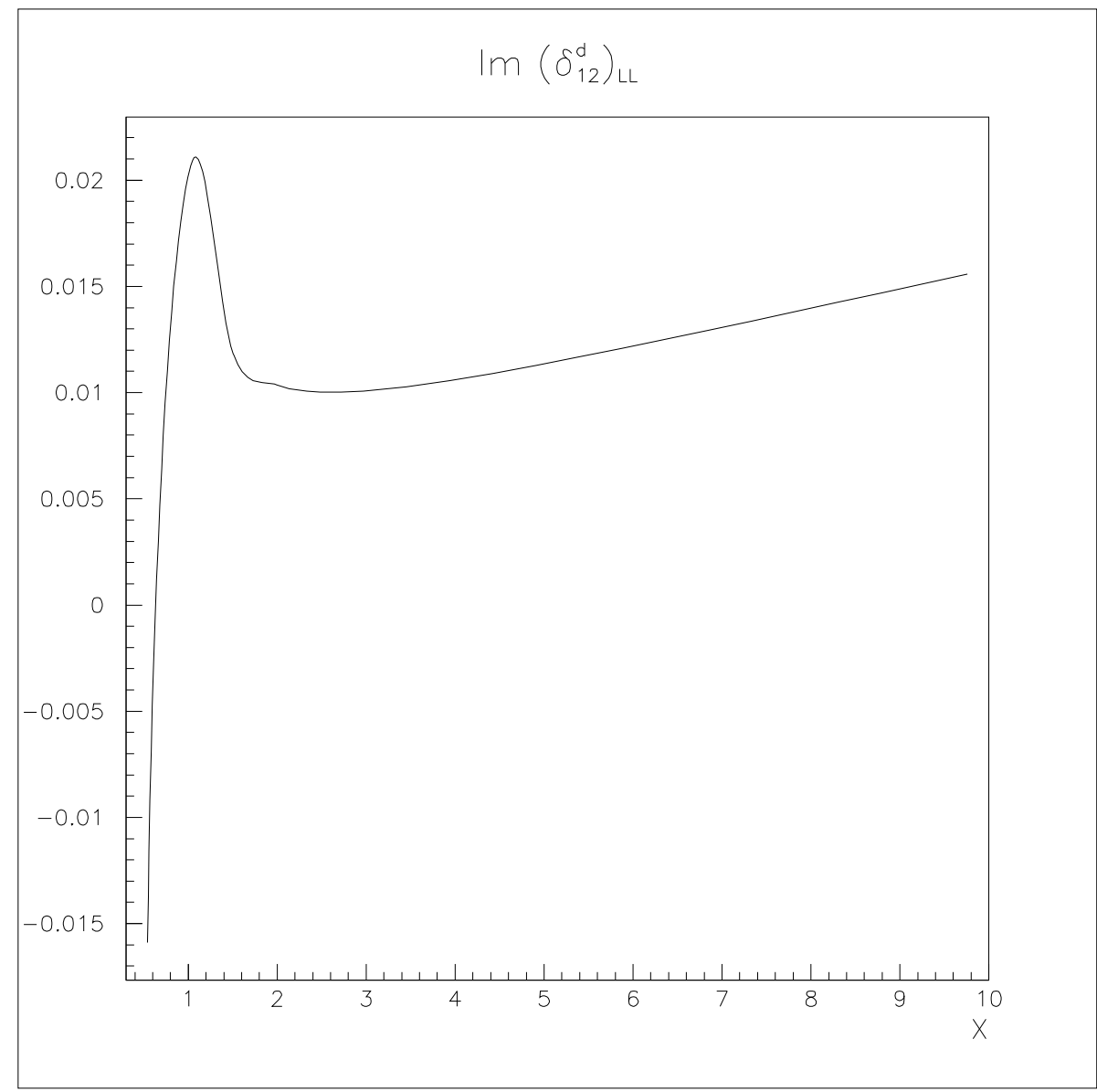

Figure 2: The $\operatorname{Im}\left(\delta_{12}^{d}\right)_{L L}$ as a function of $x=m_{\tilde{g}}^{2} / \tilde{m}^{2}$, for a squark mass $\tilde{m}=100 \mathrm{GeV}$. 


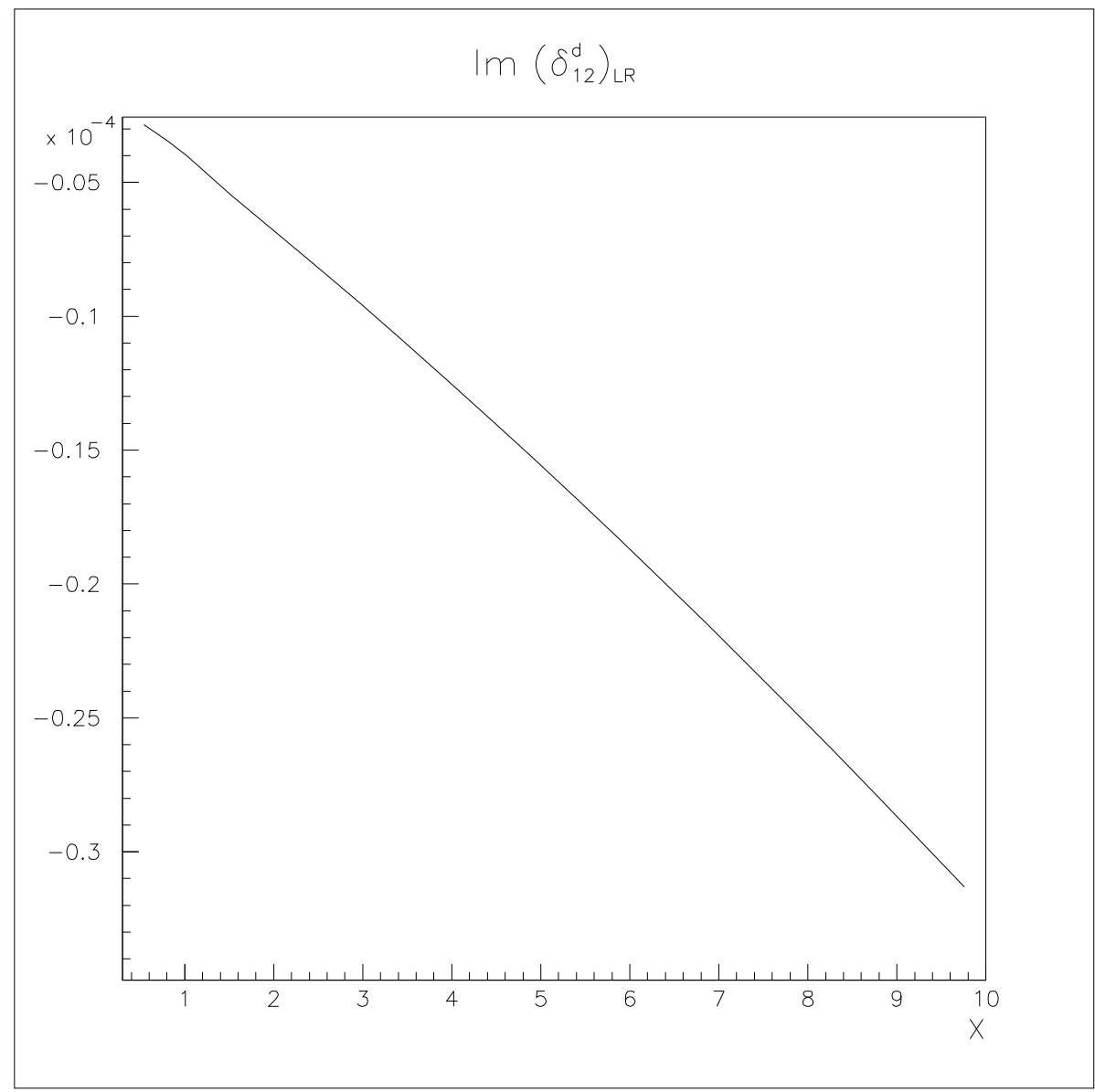

Figure 3: The $\operatorname{Im}\left(\delta_{12}^{d}\right)_{L R}$ as a function of $x=m_{\tilde{g}}^{2} / \tilde{m}^{2}$, for a squark mass $\tilde{m}=100 \mathrm{GeV}$. 